\title{
Philosophiques
}

\section{Femmes et soins dans la société post-moderne}

\section{Franchie Saillant}

Volume 21, numéro 2, automne 1994

Les femmes et la société nouvelle

URI : https://id.erudit.org/iderudit/027280ar

DOI : https://doi.org/10.7202/027280ar

Aller au sommaire du numéro

Éditeur(s)

Société de philosophie du Québec

ISSN

0316-2923 (imprimé)

1492-1391 (numérique)

Découvrir la revue

Citer ce document

Saillant, F. (1994). Femmes et soins dans la société post-moderne.

Philosophiques, 21(2), 381-392. https://doi.org/10.7202/027280ar

\section{Résumé de l'article}

L'apport des femmes à la production des soins revêt un caractère essentiel et universel tant dans l'histoire que dans la société actuelle, selon deux axes d'articulation : la protection du corps et le soutien par l'entraide et l'accompagnement. Les orientations actuelles des systèmes de santé occidentaux, comprenant une promotion idéologique de l'autonomie ayant pour conséquence la déqualification de certaines valeurs, notamment celle de l'interdépendance, compromettent l'avenir des soins dans la mesure où il n'existe pas de soin sans l'existence simultanée de la dépendance et de l'interdépendance. Le soin représente une forme de rituel d'accompagnement dans les passages de la vie, un travail de médiation.
Ce document est protégé par la loi sur le droit d'auteur. L'utilisation des services d'Érudit (y compris la reproduction) est assujettie à sa politique d'utilisation que vous pouvez consulter en ligne.

https://apropos.erudit.org/fr/usagers/politique-dutilisation/ 


\title{
Femmes et soins dans la société post-moderne*
}

\author{
par \\ Francine Saillant
}

\begin{abstract}
RESUME : L'apport des femmes à la production des soins revêt un caractère essentiel et universel tant dans l'histoire que dans la société actuelle, selon deux axes d'articulation : la protection du corps et le soutien par l'entraide et l'accompagnement. Les orientations actuelles des systemes de santé occidentaux, comprenant une promotion idéologique de l'autonomie ayant pour conséquence la déqualification de certaines valeurs, notamment celle de l'interdépendance, compromettent l'avenir des soins dans la mesure où il n'existe pas de soin sans l'existence simultanée de la dépendance et de l'interdépendance. Le soin représente une forme de rituel d'accompagnement dans les passages de la vie, un travail de médiation.
\end{abstract}

\begin{abstract}
Women's contribution to care production has been in the past, and remains in society today, of an essential and universal nature, regarding two principal concerns: care for the body and support through mutual aid and accompaniment. The current orientation of western healthcare systems, including an ideological promotion of autonomy that results in a diminishment of certain values, especially those of interdependance, compromise the future of care to the extent that there is no care without the simultaneous existence of dependance and interdependance. Care represents a sort of ritual of accompaniment in life's passage, a work of mediation.
\end{abstract}

\section{Au cour des soins : la protection du corps et l'entraide}

Le clivage que nous observons aujourd'hui entre les soins et la médecine, un clivage qui se fait en fonction du genre, est un fait somme toute assez récent dans l'histoire de l'humanité. Avant la Renaissance, cette séparation existe, mais elle est beaucoup moins nette que celle que l'on observe aujourd'hui ${ }^{\mathrm{I}}$. Plusieurs exemples sont connus, à propos de femmes soignantes

I. Voir sur cette question l'excellent ouvrage de Pat Holden et Jenny Littlewood, Anthropology and Nursing, London, Routledge, I9gi, et en particulier, le chapitre de Helen King portant sur la médecine et les soins dans la Grèce Antique. Voir aussi le récent ouvrage de Jeanne Achterberg. Woman as Healer, Shambhala, Boston, I9go.

* Le texte de cette conférence a été repris et approfondi dans « La part des femmes dans les soins de santé $\gg$, Revue intemationale d'action communautaire, vol. $28, \mathrm{n}^{\circ} 68$, 1992. p. 95-106. 
qui intégraient soins et médecine; rappellons-nous l'œuvre de Trotula de l'École de Salerne, ou encore celle de Hildegarde de Bingen ${ }^{2}$. Au cours de la Renaissance, peu à peu, dans l'Europe occidentale, on cherche à séparer magie et médecine, et à dévaluer la médecine empirique. Pour les femmes soignantes, cela aura une double conséquence. Leurs pratiques seront condamnées et associées à la médecine populaire de l'époque : nombre de femmes accusées de sorcellerie étaient, ces faits sont aujourd'hui bien connus, des femmes gardiennes du savoir des soins dans les communautés ${ }^{3}$.

C'est aussi à la même époque que les femmes furent exclues des lieux de formation de cette nouvelle médecine, savante et jugée plus rationnelle, celle des facultés, une médecine qui allait cependant s'éloigner de plus en plus de l'art de soigner. En fait, non seulement les soins que prodiguaient les femmes furent déqualifiés, comme ce fut le cas de l'ensemble de la médecine populaire, mais les femmes allaient aussi, par l'exclusion des hauts lieux du savoir, perdre le contrôle sur la connaissance du processus de soigner. Peu à peu, les soins devaient malheureusement se réduire à l'idée de service. La pensée sur les soins s'est vue enfermée et interdite. Mais, au-delà du mépris des élites savantes, ces faits n'ont pas empêché que se perpétuent les traditions de soins, certaines d'entre elles jusqu'à nos jours ${ }^{4}$. Le sort en fut autrement pour l'art de la médecine, comme on le sait, ce dernier partageant pourtant avec l'art de soigner cette origine magico-empirique. Ces processus historiques ont déterminé la séparation entre soins et médecine selon le genre, entre le cure et le care, tels que nous pouvons aujourd'hui les observer.

Selon notre propre définition, le mot « soin » signifie ici un ensemble complexe de valeurs et de symboles, de gestes et de savoirs, spécialisés ou non, susceptibles de favoriser le support, l'aide et l'accompagnement de personnes limitées, temporairement ou sur une longue période de leur existence, dans leur capacité de vivre de manière indépendante. Pour autant qu'il se peut, les soins doivent aussi permettre de favoriser le retour à l'indépendance des personnes aidées. Dans les soins, il n'y a pas d'indépendance sans la reconnaissance de l'interdépendance. Cette conception des soins reflète aussi la pensée de plusieurs théoriciennes, le plus souvent issues des professions de l'aide, qui conceptualisent depuis quelques années la notion de caring posée parfois

2. Voir Jeanne Achterberg, op. cit., chap. 6 et 7 .

3. L'ouvrage de Robert Muchembled, La sorcière au village (XVe-XVIII $\left.{ }^{\mathrm{e}}\right)$, Paris, Archives, 1979, apporte de nombreuses précisions sur la position socio-culturelle des femmes que l'on accusait de sorcellerie.

4. Voir Françoise Loux, Le jeune enfant et son corps dans la médecine traditionnelle. Paris. Flammarion, 1978. Francine Saillant, « Les recettes de médecine populaire. Pertinence anthropologique et clinique. » Anthropologie et sociétés, I4-I, 1990. p. 93II5. 
comme un équivalent de soigner, d'autres fois comme son essence ou encore comme sa condition essentielle . $^{5}$.

Mépris, dévaluation, banalisation, déqualification des savoirs des soins... au-delà de ces jugements, les soins continuent d'être surtout donnés par des femmes de toutes les sphères de la société, qui sont, par rapport à ces derniers, culturellement désignées et responsabilisées par la voie de la socialisation ${ }^{6}$. Nous ne développerons pas ici cette question déjà largement reconnue.

L'une des manières de comprendre l'importance des soins dans les différentes cultures consiste à les examiner sous l'angle de l'anthropologie. Par exemple, Leininger ${ }^{7}$ a mis en évidence, à partir d'études et d'observations effectuées dans une trentaine de cultures, le caractère universel des soins. Au-delà des différences culturelles reliées à leur expression, les soins sont partout présents. Au niveau de l'évolution et de l'adaptation, de tous les temps, les soins ont été partie de la vie; ils sont au cœur du développement des sociétés et des cultures, parce qu'ils représentent une part importante des moyens que les populations ont mis en place pour vivre et survivre. Toutes les collectivités ont dû opérer des choix en fonction des contraintes de leur environnement de manière à favoriser leur maintien et leur insertion dans des écosystèmes variés : ces choix concernent l'habitation, le vêtement, l'alimentation, les styles de vie, la technologie; ces choix sont aussi de l'ordre des divers moyens de prévenir la maladie, ou de la pallier, mais aussi d'entretenir la vie dans le sens le plus large 8 .

On commence de plus en plus à reconnaître l'apport des femmes aux divers systèmes de médecine du monde, par le biais d'une meilleure compréhension de leur contribution à la sélection, à la cueillette, à la conservation et à la préparation des plantes médicinales ${ }^{9}$. Par rapport à l'évolution, les femmes

5. Voir notamment Marie-Françoise Collières, « Invisible Care and Invisible Women as Health Care Providers », International Journal of Nursing Studies, 23 (2), 1986, p. 95II2. Madeleine Leininger, Caring, an essentiel Human Need, Proceedings of the Three National Caring Conferences, Détroit, Wayne State University Press, 1988. Francine Saillant, «Les soins en péril : entre la nécessité et l'exclusion », Recherches féministes, vol. 4 (I), I99I, p. II-30. Janice M. Morse, Shirley M. Solberg, Wendy L. Neander, Joan L. Botorff, Joy L. Johnson, « Concepts of caring and caring as a concept », Advance Nursing Scicncc, vol. i3 (I), 1990, P. I-14.

6. Nous avons déjà développé ailleurs cette question, $c f$. Francine Saillant, op. cit.

7. Madeleine Leininger, op. cit.

8. Voir sur ces questions : Madeleine Leininger, Transcultural Nursing, Concepts, Theories, Practices, New York, Wiley \& sons, 1978. Marie-Françoise Collières, Promouvoir la vie. De la pratique des femmes soignantes aux soins infirmiers, Paris, Interéditions, I985.

9. Sharon A. Sharp, « Folk Medicine Practices : Women as Keepers and Carriers of Knowledge », Women's Studies Forum, vol. 9, no 3. 1986, p. 243-249. Francine Saillant, « Pratiques et savoirs des femmes dans l'univers ethnomédical québécois », Canadian folklore canadien, vol I4 (I), 1992 : P. 47-72. 
ont été au premier plan dans les activités de cueillette, contribuant à nourrir mais aussi à soigner; bien des plantes connues pour leurs propriétés médicinales ont d'abord été sélectionnées pour des fins alimentaires ${ }^{\text {IO }}$. Les soins généralement développés par les femmes sont enchassés dans les activités de la vie quotidienne (agriculture, alimentation); ils favorisent entre autres la protection du corps. Par exemple, dans la société traditionnelle québécoise, les femmes préparaient des tisanes à base de résineux que l'on devait prendre tout l'hiver pour prévenir les maladies du froid. Cette pratique eut longtemps une valeur adaptative, si l'on tient compte du contenu élevé en vitamines $C$ de ces tisanes ${ }^{\text {II }}$. Nombre de recettes médicinales ou recettes dites de grand-mère, qui furent jusqu'à la fin du XIXe siècle la principale ressource thérapeutique des familles québécoises, étaient fabriquées non seulement à partir de plantes médicinales, mais aussi à partir d'ingrédients de l'alimentation quotidienne, selon le principe : ce qui nourrit, protège et reconstitue le corps, peut aussi guériri $^{12}$.

Les soins sont reliés à la survie des populations non seulement en ce qui concerne la protection du corps, en regard des savoirs et pratiques de la vie quotidienne de nature préventive, curative et palliative, mais aussi par rapport au support social qu'ils peuvent générer.

De manière plus théorique, le support social serait la somme de tous les échanges, sociaux, instrumentaux et émotionnels existant entre les individus; ces échanges font qu'un individu se percevra ou non comme un objet de valeur face aux autres significatifs ${ }^{\mathrm{I}}$. Le support social, permettrait en fait une plus grande sécurité par rapportà l'interdépendance. Sans le support, nous sommes forcés à plus d'indépendance et de solitude. Le fait de ressentir qu'on prend soin de Soi implique de la part de l'Autre une part nécessaire d'empathie. Le support social c'est souvent le fil connecteur qui donne le sens à la vie. Les connections aux autres significatifs permettent les liens, le maintien de l'identité sociale, le maintien de la réalité; la personne qui est supportée sait quel sens donner à sa vie, et qui lui donne du confort au besoin. Les « fils connecteurs » sont la plupart du temps de nature informelle et subjective.

Comme l'ont souligné Pillisuk et Parks ${ }^{14}$, la promotion du support social peut permettre la promotion de relations de cohésion et de réciprocité, et les

Io. Pour plus de précision sur le rôle des femmes dans l'évolution et sur leur apport dans la sélection des plantes utiles à des fins alimentaires et médicinales, voir Adrienne L. Zilhman, «Woman the Gatherer : the Role of Women in Early Hominid Evolution », dans Sandra Morgan, Gender and Anthropology, Washington, American Anthropological Association, 1989, p. 21-40.

II. Francine Saillant, « Le rhume et la grippe. Recettes québécoises de médecine populaire », Ethnologie française, vol. 4 (I), 199ı, p. II-30.

12. Francine Saillant, « Pratiques et savoirs des femmes... », op.cit.

13. Mark Pillisuk, Susan H. Parks, Social Network ands human survival, London, University Press of New England, 1986. p. 17.

14. Ibid., chap. I. 
valeurs de cohésion et de réciprocité sont au cœur de la pratique des soins. Dans la société québécoise, les exemples de pratiques de soins illustrant l'importance du support social sont nombreux. Parmi d'autres, retenons celui des infirmières de colonies, c'est-à-dire de ces femmes qui sont chez nous les pionnières de la santé communautaire. Ces infirmières, que le grand public a connues sous la figure de la Blanche Pronovost du roman Les filles de Caleb, furent engagées au début du siècle par le ministère des colonies, et constituaient la principale ressource socio-sanitaire des populations qui s'installaient dans des régions éloignées. Non seulement prodiguaient-elles des soins d'urgence, (entendre ici des services médicaux), mais elles se présentaient comme de véritables agents de cohésion et de développement communautaire. Elles prêtaient et donnaient de l'argent, elles conseillaient, elles étaient de toutes les fêtes et de tous les malheurs. Elles étaient à la fois maille et pivot du tissu social ${ }^{15}$.

En résumé, il est possible d'appréhender l'importance des soins selon deux axes interreliés. Le premier, celui de la protection du corps, favorise l'entretien de la vie et la survie; le deuxième, celui de l'entraide, apparaît un ingrédient essentiel du développement des communautés. Nous affirmons que les deux axes sont interreliés, c'est-à-dire connectés selon un rapport de type holiste. Les soins du maternage réunissent protection et support; il en est de même des soins à l'adulte âgé fragilisé que donne une fille à sa mère ou à son père, ou encore des soins spécialisés de l'infirmière à domicile à l'individu mourant d'un cancer en phase terminale. Ces axes organisateurs du phénomène soin permettent de préciser ce que nous apprend l'étymologie du mot « soin », c'est-à-dire se préoccuper de, souci, sollicitude. Par rapport aux valeurs qui sous-tendent ces pratiques et savoirs, on note la place centrale accordée à l' Autre, et l'acceptation des réalités indissociables de la dépendance et de l'interdépendance.

\section{l'autonomie tous azimuts et les pièges d'une société sans soins}

Depuis les trente dernières années, dans l'ensemble des pays occidentaux, nous assistons à la transformation profonde du paysage socio-sanitaire, autrefois caractérisé par la prévalence des maladies infectieuses. Dans les pays occidentaux, cette transformation correspond à une configuration de conditions sociales et de maladies prévalentes, incluant, de l'avis d'une majorité d'experts

15. L'histoire de ces infirmières et leur contribution au développement sociosanitaire du Québec restent entièrement à connaître. Une recherche en cours, sous la direction de Nicole Rousseau, en collaboration avec Johanne Daigle et Francine Saillant, permettra de décrire et analyser, selon une perspective historique, la contribution de ces femmes au maintien et au développement des populations des régions isolées du Québec. 
I) le vieillissement de la population et la création du quatrième âge; 2) la prépondérance des maladies chroniques, donc la possibilité pour de nombreux individus de vivre plusieurs années de leur vie en souffrant d'une maladie ou d'une condition impliquant un déficit fonctionnel et une perte quant à la qualité de vie et à l'autonomie; 3 ) la souffrance sociale reliée à la pauvreté, à l'isolement, à la marginalité et à la violence subie par des portions de plus en plus étendues de population; 4) l'arrivée d'une nouvelle épidémie, le SIDA, associant ici, pour les individus qui en sont atteints, l'état de chronicité, la marginalisation, la souffrance physique et morale, et souvent, la pauvreté. En ce sens, nos sociétés font face à une modification des images de la maladie et de la souffrance et des diverses formes de dépendance qui en découlent.

Au cours de la même période, et de façon plus intense depuis les dix dernières années, nous avons assisté au démantèlement de l'État Providence, un phénomène ayant affecté largement les systèmes de santé des pays occidentaux, qui font face à une crise par rapport à leur développement. Pour que la population ait accès véritablement à ce système de santé, elle doit aujourd'hui payer de plus en plus cher, en raison des transformations du paysage socio-sanitaire, mais aussi en raison des technologies complexes que requiert la biomédecine ${ }^{\mathrm{T} 6}$. De plus en plus de gens considèrent que, bien que la santé doive toujours être considérée comme un droit, la biomédecine ne saurait être le moteur essentiel du développement des systèmes de santé, d'autant plus que les traitements médicaux ne répondent pas à eux seuls aux besoins, largement déterminés par des facteurs sociaux, culturels, politiques et économiques. Le droit à la santé devra s'appuyer non seulement sur la biomédecine et ses ramifications, mais surtout sur des politiques de promotion de la santé axées sur le développement communautaire, l'auto-santé et l'aide mutuelle. A noter que nous retrouvons ici, sous une terminologie qui masque la question du genre, les axes de protection du corps et d'entraide que nous considérions précédemment comme les axes organisateurs de la pratique et des savoirs des soins.

Parmi les États modernes se situant à la pointe des réajustements nécessités par ces transformations, on compte le Canada. Plusieurs textes récents, inspirés de l'OMS ${ }^{17}$, rassemblent les grandes lignes du virage en train de s'opérer. Citons à ce titre la Charte d'Ottawa et le rapport $\mathrm{Epp}^{\mathrm{I} 8}$, lesquels convergent vers une seule et même idée : il faut produire la santé à moindre coût, baser nos interventions sur la communauté et la participation, favoriser

16. Nous employons le mot «biomédecine » pour désigner la médecine scientifique occidentale telle qu'elle s'est développée depuis l'après-guerre.

17. Organisation mondiale de la santé, Promotion de la santé, une base de discussion sur le concept et les principes, Copenhage, OMS-Europe, 1984.

18. J. Epp, Achieving Health for All : a Framework for Health Promotion, Ottawa, Minister of Supply and Services, 1986, The Ottawa Charter of Health Promotion, Health Promotion, an International Journal, I (4), 1986, p. III-v. 
l'autonomie, la responsabilité et l'appropriation, mettre en place des environnements sains, générateurs des conditions favorables à des modes de vie sains. Pour les individus et les communautés, sains ou malades, le message est le même : l'État veut produire des individus autonomes et producteurs, qui seront le moins dépendants possible des ressources humaines et économiques de leur environnement.

Par ailleurs, ce nouveau système de santé se construit sur un fond social précis, que nous qualifierons de post-moderne. On réfère le plus souvent à la société post-moderne en soulignant la montée des valeurs reliées à l'hyperindividualisme. Deux thèses s'affrontent le plus souvent, que nous classerons en les qualifiant d'hypothèse de la société froide et d'hypothèse de la société chaude. On associe le déni de la mort, de la souffrance et du handicap, l'anomie et la désintégration sociale des communautés de base à la société froide ${ }^{19}$. Dans la société chaude, parfois qualifiée de société tribale, les nouvelles familles se substitueraient à la famille nucléaire. Des solidarités nouvelles se construiraient sur les réseaux d'entraide, de plus en plus nombreux et spécifiques, générateurs d'identités nouvelles et foisonnantes, et des nouvelles formes associatives s'élaboreraient à partir de l'affect et du besoin de proximitée ${ }^{\mathrm{O}}$. La réalité dépasse probablement ces deux caricatures.

La souffrance, les états-limites, la maladie sont susceptibles de favoriser l'expression plus ou moins ouverte du besoin de l'Autre, et de générer, à des degrés divers, la dépendance. Cela peut conduire à des situations d'isolement et de rupture sociale caractéristiques de la société froide; nous reconnaissons ici la «lady bag » du centre-ville. Cela peut aussi générer les identités émergentes de la société chaude; on retrouve ici l'ex-psychiatrisé membre d'une association d'entraide et luttant pour ses droits. En fait, dans cette société post-moderne, ces deux réalités co-existent, exprimant la tension entre les valeurs d'interdépendance et d'hyperindividualisme. Ultimement, dans la société post-moderne, on demande aux indigents, aux gens qui souffrent et qui sont susceptibles de demander de l'aide, d'être le plus autonome possible. Dans cette société de prise en charge collective, et au niveau des politiques de santé, protection et entraide coexistent dans le but de promouvoir le développement maximal de l'autonomie. Il y a bien sûr les coûts sociaux et économiques que l'on cherche à éviter, mais il y a plus : l'idée de devoir supporter un ou des êtres dépendants devient de plus en plus inacceptable pour l'État, et pour la société.

L'individu aux prises avec une condition sociale limite (SIDA, Alzheimer...) se fait dire qu'il doit compter sur les autres proches et sur son

19. Voir par exemple Gilles Lipovetsky, Lère du vide. Essai surl'individualisme contemporain, Paris, Gallimard, 1983.

20. Voir par exemple Michel Maffesoli, Le déclin de l'individualisme dans les sociétés de masse, Paris, Méridiens Klicksieck, 1988. 
entourage, et sur lui-même, pour vivre et survivre. Dans cet esprit, la promotion de l'autonomie, survalorisée, prend un caractère normatif et idéologique. Les individus jugés non autonomes, fragilisés par une condition ou une souffrance particulière, doivent « apprendre à vivre avec leur maladie », « apprendre à mourir », « gérer leur handicap », etc. Ils représentent autant d'identités parcellaires et éclatées, autant d'images de la souffrance à effacer. L'auto-santé et l'aide mutuelle, autrefois si menaçantes face au professionnalisme et au pouvoir médical, se transforment dans le contexte post-moderne en panacées pour l'État, qui cherche par tous les moyens à limiter ses dépenses et à se décharger de ses « poids lourds». Le sens de ces notions se trouve ici étrangement perverti. Par rapport aux valeurs centrales des soins, l'indépendance prend le pas sur l'interdépendance.

$\mathrm{Au}$ cours des dernières années, tous les services de santé générés par l'État ont été suspectés de coûter cher : tout est devenu de trop, sauf bien sûr, la technologie biomédicale et les médecins. On a diminué les temps de séjour, augmenté considérablement le ratio bénéficiaire-soignante, créé toutes sortes de services, changeant les habitudes hospitalières. Tout le monde a dû retourner chez soi : les vieux, les agonisants, les fous. Dans l'ensemble, les soins formels ont pris une place différente, utilisés au prorata des besoins quotidiens; le réseau communautaire et la famille (c'est-à-dire des femmes) ont pallié les trous.

Dans la société actuelle, des milliers de femmes sont présentes à tous les niveaux de la société en ce qui concerne les soins de santé: elles sont le moteur de la culture des soins, tant dans le réseau des services de santé que dans le réseau communautaire et dans la famille, agissant dans le secteur formel et dans le secteur informel. Le travail des soins est au système de santé ce que le travail domestique est à l'économie marchande : nécessaire et essentiel mais sans valeur et banalisé2 ${ }^{21}$.

Le développement des systèmes de santé occidentaux s'est par le passé appuyé sur la santé comme droit universel, sur le système biomédical, c'est-àdire sur son pouvoir, son prestige et ses liens obligés avec l'économie marchande, mais aussi sur l'acquis des savoirs et des pratiques des soins prodigués surtout par des femmes, professionnelles ou non, des soins jugés essentiels mais sans valeur, la valeur pouvant ici être marchande, intellectuelle, symbolique, thérapeutique, etc.

Dans la critique globale de l'État Providence et du système de santé qui en était caractéristique, les soins sont fréquemment assimilés aux services de santé, sans distinction de leur nature et de leur spécificité. Ils prennent ici le sens du service, le health care des anglo-saxons. Nous venant aussi des pays anglo-saxons, la séparation conceptuelle entre le cure et le care, si largement

2I. Cette idée est largement développée dans Francine Saillant, « Les soins en péril... », op. cit. 
acceptée, contribue à sa façon à entretenir la confusion et la banalisation. Le care peut se réduire à la notion de véhicule du traitement, c'est le soin dont on entoure la personne en la traitant (c'est l'infirmière qui fait passer la pilule !), ou encore, plus récemment, il prend la forme du fardeau des aidants, c'est le burden of care.

Dans le système de santé qui se construit, les soins sont entraînés dans le rouleau compresseur des réductions budgétaires; c'est le facteur humain qui coûte trop cher dans les organisations. Ils sont parfois gentiment maquillés pour permettre l'humanisation de la médecine-spectacle, ce qui fait que l'on peut vendre la médecine telle qu'elle est. Ils alourdissent la vie des familles et de toutes les aidantes : c'est le burden of care, le fardeau qui épuise, c'est le poids du «dumping » médical et de la «désins $»^{22}$... Ils sont tour à tour trop, rien, pas assez... une histoire trop bien connue. Le système de santé des années ig9o prolonge cette histoire, mais de façon encore plus pernicieuse : non seulement compte-t-il sur ces soins, l'apport naturel et attendu des femmes, mais il les dénature, en rendant l'Autre, psychotique, alzheimer, errant, sidéen, coupable de sa dépendance.

\section{Soigner : à propos du passage et du don}

Dans le système de santé qui se construit en relation avec le paysage socio-sanitaire des années 1990, les personnes qui agissent au cœur des soins, qu'elles soient professionnelles ou non professionnelles, ont de plus à composer avec des situations limites. Dans le domaine des soins à domicile, par exemple, cela signifie être en contact avec des personnes assaillies par des démons qui ne supportent pas d'être touchées ou approchées, avec des vieillards sans mémoire, des quadraplégiques, des incontinents, bref, des gens qui ont perdu, à un titre ou un autre, le contrôle d'une partie de leur corpsesprit. Il s'agit de personnes déclassées ou sans statut, ou encore inclassables par rapport à un statut.

Dans un récent article, Littlewood ${ }^{23}$ aborde cette question en faisant ressortir que le travail des soins consiste, en fait, à accompagner cette catégorie de personnes dans leur position ambiguë de liminale. Selon nous, ces aspects du travail des soins particularisent, sur le plan individuel et dans un microenvironnement, les axes organisateurs de la protection et de l'entraide. J'explorerai ici le thème de la perte de contrôle du corps. Je reprendrai, dans ses grandes lignes, l'article de Littlewood et Holden.

La personne qui ne maîtrise pas son corps entre dans une catégorie « grise » au plan de son statut, elle est polluée, impropre. La maîtrise du corps par le contrôle de la propreté est un signe de l'entrée dans la vie humaine.

22. Diminutif pour désigner la désinstitutionalisation.

23. Jenny Littlewood, « Care and Ambiguity : towards a Concepts of Nursing », dans Holden et Littlewood, op. cit., p. I70-189. 
Avant son entrée au monde, le bébé est entièrement dépendant de sa mère, il est inachevé. Pour un adulte, le retour vers cet état de non-contrôle signifie le retour vers la dépendance, vers la régression, vers une forme de vie enfantine. Ne disait-on pas autrefois d'un vieillard sénile: « Il est retombé en enfance »? La femme soignante, en contact avec cette réalité, partage les émotions du malade et de ses proches qui vivent l'humiliation dans cette perte de contrôle. En partageant la pollution du corps, de l'adulte en perte de contrôle, la soignante entre dans le monde de cet Autre; elle partage aussi la réalité d'être sans statut, ou inclassable par rapport à un statut. Dans beaucoup de sociétés, les soins et les soins infirmiers en particulier sont d'ailleurs culturellement associés à la pollution et à la saleté. En Inde, pendant longtemps, seules les femmes de la caste des intouchables étaient assignées à la manipulation des déchets du corps malade ${ }^{24}$. Na-t-on pas souvent qualifié les infirmières avec mépris, les qualifiant de « videuses de bassines », pour parler de la partie de leur tâche qui est de transiger avec les déchets d'un corps provisoirement ou totalement en perte de contrôle?

La division du travail dans une unité de soins peut être celle de la manière dont on se partage dans la réalité le sale et le propre. Comme le fait remarquer Littlewood ${ }^{25}$ :

The traditional association of women with pollution, low status work, division of labour within the ward has separated off one form of polluantsbodily secretions and excretions-from another-dust, spillage from flower vases, and so forth. Nurses are expected to clear up urine, faeces, and vomit. These are « sick dirt », so highly polluting that they would not be removed by the domestics.

Par rapport aux personnes ayant perdu le contrôle de leur corps, le travail des soins devient une forme de présence protectrice et de support en fonction de cet état liminal et ambigu entraîné par la perte de contrôle de son corps. La soignante, par son rôle d'intermédiaire entre le monde du patient, de la maladie, et le monde extérieur, « normalisé », permet l'actualisation d'un espace permissif qui autorise la saleté et la pollution. La présence de la soignante symbolise la possibilité de ce non-contrôle, le sanctionne en quelque sorte.

Cette question de la relation au passage a son corollaire, celui de la médiation. Des études récentes en anthropologie de la santé ${ }^{26}$ soulignent le fait que les femmes se situent souvent dans des positions de marginalité, et dans les sociétés traditionnelles, dans différents mondes (ce monde, le monde des ancêtres). Ce sont elles qui donnent réellement naissance, à travers leurs

24. Voir Geeta Somjee, «Social Change in the Nursing Profession in India », dans Holden et Littlewood, op. cit., p. 31-55.

25. Jenny Littlewood, op. cit., p. 125.

26. Carol Shepherd McClain, Women as Healers. Cross cultural Perspectives, New Brunswick, Nutgers University Press, 1989, p. I-19. 
corps, et font passer de la non-vie à la vie, (elles et la sage-femme), tandis qu'elles font aussi passer de la vie à la mort, dans les rites funéraires (le phénomène des mourners). Elles sont celles qui relient symboliquement, par un travail de soins, les passages entre la vie et la mort.

Dans la société moderne, où l'on assiste à la reconfiguration des oppositions vie-mort, les états bio-culturels intermédiaires se multiplient. Du côté de la naissance, on assiste à un allongement des possibilités de vie pour les prématurés; par ailleurs le temps du mourir est aujourd'hui très long, si l'on compare notre époque aux périodes historiques des grandes épidémies. La prévalence des maladies chroniques a aussi introduit le statut de malade chronique, multipliant encore là les statuts intermédiaires, cette fois-ci par rapport à la maladie. Plus que jamais, le travail des soins consiste à accompagner les personnes dans ces états-limites, dans ces zones intermédiaires de la vie, là où les statuts sont perdus, inexistants ou ambigus. Ce travail nécessite une relation au don et à l'Autre, à la reconnaissance de la dépendance et de l'interdépendance.

Le don est en fait au cœur de l'éthique des soins, une éthique que Gilligan et Noddings ont appellée la morale de la sollicitude (ethic of caring). Bien résumée par Bureau ${ }^{27}$, cette éthique

[...] trouve son fondement privilégié dans le fait que l'être humain est « un être avec les autres » un « être en relation aux autres » et, de ce fait, responsable des autres. Pour la morale de la sollicitude, l'Autre est considéré en particulier, C'est toujours cet Autre, avec un tel visage, vivant telle situation, à sa façon bien particulière; l' Autre est ainsi vu comme différent de soi; c'est celui ou celle à qui il faut penser, à qui il faut « répondre », dont on doit s'occuper.

De nombreux exemples dans une récente recherche conduite par Carpentier ${ }^{28}$ illustrent comment, chez des infirmières qu'elle a interviewées, intervient cette relation particulière des soins à la sollicitude et au don. Comme elle le dit elle-même :

Leur engagement est à ce point important que dans des départements comme celui d'oncologie où plusieurs patients vivent la phase terminale, il n'est pas rare de voir des infirmières faire des achats pour leur malade, leur confectionner des vêtements, et lorsque la mort arrive, elles iront même parfois jusqu'à accompagner la famille au salon funéraire.

Carpentier donne aussi l'exemple d'infirmières amenées à faire sauter les barrières de l'asepsie envers des malades atteints de sida; citant l'une d'entre elles $^{29}$ : « On ne peut refuser à un être humain qui va mourir un dernier contact humain malgré le risque d'infection. On fait attention, c'est tout. »

27. Lucille Roy Bureau, «Femmes et développement moral : la fin d'un mythe », Critique féministe des disciplines, Cahiers du Gremf, vol. 3 (2I), p. 35. Ce texte présente d'une manière claire et synthétique. les thèses de Gilligan et Noddings.

28. Marie-Claire Carpentier Roy, Corps et âme, psychopathologie du travail infirmier, Montréal, Liber, I99i, p. 70.

29. Marie-Claire Carpentier Roy, op. cit., p. 92. 
Ces exemples renforcent le point de vue que nous développions à propos du problème du passage. Transigeant dans les états limites, en bref dans ces lieux de passage, les soignantes servent ni plus ni moins de connecteurs dans un univers qui cherche plutôt à séparer, à diviser et à hiérarchiser. Connexion entre le propre et le sale, connexion entre le contrôle et le non-contrôle, connexion entre la vie et la mort.

\section{Conclusion}

De par les savoirs qu'ils recèlent, de par leur importance pour la vie et le développement des individus et des communautés, et en regard des valeurs porteuses qu'on leur reconnaît, les soins méritent plus que le sort que la société leur fait. Post-moderne ou non, le sort que l'on réserve aux soins est celui de la société patriarcale : mépris, dévaluation, déqualification, oubli. Que les femmes soient massivement impliquées dans le travail des soins, dans l'obligation à ce travail, est contestable et inacceptable. Mais que ce travail, ces savoirs et ces pratiques se perdent dans une nébuleuse infinie, sans nom, et dans l'indignité, l'est encore plus.

Peut-être pourrions-nous jeter un autre regard sur les soins; ce système de santé « autre » sur lequel le pouvoir sait si bien compter, quel est-il ? Connaît-on vraiment de quoi il est fait ? Sommes-nous capables de percevoir le savoir dans les gestes de soins?

Ce qui est en fait rejeté entre les mains des femmes, c'est justement ce dont le pouvoir ne veut pas : la mort, la souffrance, la demi-vie, la folie; c'est l'intolérance, la maladie sociale. C'est le corps qui pue, hurle, s'aggrippe. On peut juger et dire : cela est impossible et injuste, les femmes ne peuvent être collectivement responsabilisées pour cela, ce qui est vrai. Mais avant de rejeter du revers de la main les soins, parce qu'idéologiquement trop marqués, une autre question se doit d'être posée, en prenant pour point de départ les valeurs qu'ils portent. Une hypothèse se dessine.

La société post-moderne est une société sans Autre. On veut des malades gestionnaires, bien organisés, autonomes, scientifiquement contrôlés. Pas de bave, pas de gros mots. Que des silences feutrés, c'est l'idéal de l'autonomie. La «lady bag » du centre-ville est autonome.

Le système de santé des soins, invisible et invisibilisé, prend pour point de départ l'acceptation de l'altérité. Les soins, librement choisis, non confinés à un genre, à une classe ou à une profession, parfaitement visibles et valorisés, pourraient être à la base d'une nouvelle éthique socio-sanitaire, d'une politique de la santé. Mais cela, actuellement, ce sont les femmes, et toutes celles qui soignent, qui peuvent le dire. 